\title{
Equipamento de baixo custo para monitoramento de pressões em sistemas de abastecimento de água
}

\author{
Low cost equipment for monitoring pressures in water supply systems
}

\author{
Renato Leandro Beregula** ${ }^{1}$, Fernando Rodrigues da Silva² ${ }^{\circledR}$
}

\begin{abstract}
RESUMO
A importância de se monitorar as pressões no sistema de abastecimento de água aliada ao alto custo de implementação e manutenção de dispositivos capazes disso corroboraramao objetivo deste estudo. Sendo assim, buscou-se desenvolver um equipamento de baixo custo para monitorar a pressão da água. Para isso, produziu-se 15 MPR dataloggers, custando R\$208,15 cada, que foram aferidos com um equipamento comercial. Os equipamentos foram instalados em residências de distintos perfis de consumo e altitudes do município de Lucas do Rio Verde, no estado de Mato Grosso, por 43 dias. De modo geral, os resultados obtidos foram satisfatórios, promissores e demonstraram o equipamento robusto e preciso, com R² de 0,9986 quando comparado com o equipamento comercial, além de ter sido possível avaliar o sistema de abastecimento da cidade e as intervenções necessárias.
\end{abstract}

Palavras-chave: hidráulica; rede de abastecimento; hardware livre; datalogger

\begin{abstract}
The importance of monitoring the pressures in the water supply system combined with the high cost of implementation and maintenance of devices capable of this, corroborated the objective of this study. Therefore, we seek to develop low-cost equipment to measure water pressure. For this, 15 MPR dataloggers were produced, costing R\$208.15 each, calibrated with a commercial equipment. The equipment were installed in residences with different consumptions profiles and altitudes in the municipality of Lucas do Rio Verde, Mato Grosso, for 43 days. The results obtained were satisfied, promising, and showed that the equipment is robust and precise with a $\mathrm{R}^{2}$ of 0.9986 when compared with the commercial equipment, in addition to being possible to evaluate the city's supply system and the necessary interventions.
\end{abstract}

Keywords: hydraulics; water supply network; open-source hardware; datalogger.

\section{INTRODUÇÃO}

O sistema de abastecimento de água tem seu funcionamento analisado sob a perspectiva de fatores físicos e hidráulicos. Nesse sentido, os fatores físicos podem ser observados por meio da ocorrência de rompimentos, vazamentos ou mesmo entupimentos. Em relação aos fatores hidráulicos, o funcionamento do sistema deve ser avaliado de acordo com a eficácia da distribuição das vazões e das pressões. Em outras palavras, analisa-se a capacidade do sistema em fornecer o volume de água demandado (TSUTIYA, 2006).

As pressões e as vazões são grandezas que variam com grande frequência no sistema de abastecimento de água, principalmente em função das oscilações de sua demanda durante o dia, do perfil socioeconômico, da localização, do clima, entre outros fatores (DIAS; MARTINEZ; LIBÂNIO, 2010), inferindo em dificuldades na determinação dessas variáveis. Diversos estudos abordam a importância do acompanhamento e do controle das pressões no sistema de abastecimento de água, apresentando-se como uma das principais opções técnicas para incremento da eficiência hidráulica e energética, além da criação de um banco de dados para possíveis modelagens em relação às pressões e aos vazamentos (SOARES et al., 2004; FORMIGA; CHAUDHRY, 2008; CAMPISANO; MODICA; VETRANO, 2012; FECAROTTA et al., 2015; SARBU, 2016; MOURA et al., 2018).

O monitoramento das pressões por dispositivos de coleta e armazenamento de dados nas redes de abastecimento de água pode ser considerado parte integrante da automação do sistema e base para tomada de decisão. Desse modo, um dos primeiros trabalhos descritos na literatura científica visando ao controle dos sistemas de abastecimento é de Fallside e Perry (1975). No Brasil, Trojan e Marçal (2007)

'Departamento de Engenharia Sanitária e Ambiental, Universidade Federal de Mato Grosso - Cuiabá (MT), Brasil.

²Departamento de Engenharia Sanitária e Ambiental, Programa de Pós-Graduação em Saneamento, Meio Ambiente e Recursos Hídricos, Universidade Federal de Minas Gerais - Belo Horizonte (MG), Brasil.

*Autor correspondente: renato_beregula@hotmail.com

Recebido: 14/03/2018 - Aceito: 19/08/2019 - Reg. Abes: 192896 
monitoraram as vazões e as pressões de um sistema de abastecimento de água a fim de reduzir os índices de perdas de água, reduzir a pressão média e criar um banco de dados. Além disso, Bezerra, Silva e Gomes (2012) desenvolveram, em escala laboratorial, uma ferramenta capaz de monitorar e controlar o plano piezométrico do sistema de distribuição de água por conversores de frequência e válvulas redutoras de pressão.

De maneira geral, observa-se que diversas pesquisas têm sido desenvolvidas a fim de monitorar parte do sistema de distribuição de água, baseando-se no princípio de automação e coletas de dados por dataloggers. Willis et al. (2013) desenvolveram um datalogger para observar o consumo de água e relacionar com os fatores sociodemográficos. Gurung et al. $(2014 ; 2015)$ utilizaram dataloggers comerciais para acompanhar o consumo de água individual de um grupo amostral para desenvolvimento de uma modelagem estatística. Nesse mesmo sentido, Guragai et al. (2018) também realizaram a medição do consumo de água residencial por um datalogger comercial em um sistema de abastecimento intermitente. No entanto, poucas pesquisas utilizam dataloggers comerciais ou desenvolvem protótipos para monitoramento específico das pressões nos sistemas de distribuição de água e suas possíveis intervenções.

Os fatores de altos custos de instalação e manutenção dos sistemas capazes de coletar e armazenar os dados ambientais são comumente apresentados como limitantes no emprego desses dispositivos, principalmente nos países em desenvolvimento (BARROS FILHO et al., 2018). Nesse sentido, Sadler, Ames e Khattar (2016) apresentaram um encadeamento lógico para o desenvolvimento de equipamentos dataloggers para aquisição e compartilhamento de dados ambientais baseados no uso de padrões de softwares de fontes livres e módulos eletrônicos de baixo custo. O sistema proposto foi baseado em sensores de medição de temperatura e umidade acoplados a um coletor de dados e bateria autônoma, tendo sido demonstrada a possibilidade de adaptação para outros sensores de interesse.

Em relação ao desenvolvimento desses equipamentos para monitoramento de variáveis ambientais, os avanços na área de hardware livres, tais como Arduino e Raspbery Pi, vêm sendo aplicados em pequenos computadores e microcontroladores de massas, inferindo na redução de custo de equipamento em muitas áreas técnicas. Mais especificamente, pesquisas vêm sendo realizadas com hardware livres no monitoramento de parâmetros, como uso de latrinas, profundidade de neve, solo, umidade, qualidade do ar, ambientes marinhos e até níveis de patogenicidade (LUNDQUIST; LOTT, 2008; CLASEN et al., 2012; TREVATHAN et al., 2012; FEDI et al., 2013; BAKER, 2014; MITCHELL; CHUA; SON, 2014; SADLER; AMES; KHATTAR, 2016).

Sob a perspectiva que tange à importância do monitoramento das pressões nas redes de abastecimento de água, aos custos de aquisição e instalação dos componentes comerciais para tal monitoramento e à carência do aporte técnico-científico no desenvolvimento de opções de baixo custo, a presente pesquisa teve por objetivo desenvolver um equipamento de medição de pressões hidráulicas no abastecimento de água não oneroso, aplicando-o no monitoramento das pressões em residências do município de Lucas do Rio Verde, no estado de Mato Grosso.

\section{METODOLOGIA}

O presente trabalho desenvolveu 15 dispositivos medidores de pressão hidráulica para monitoramento em redes de abastecimento, denominados MPR datalogger, de baixo custo. Portanto, buscando-se acompanhar o desempenho dos dispositivos e o comportamento da pressão da água em uma rede de abastecimento real, os equipamentos desenvolvidos foram instalados em 15 residências distribuídas pelo município de Lucas do Rio Verde, Mato Grosso.

\section{Hardware}

Os critérios de seleção dos componentes do sistema de medição e registro de dados da pressão da água foram tomados visando ao desenvolvimento de um dispositivo que fosse autônomo, de baixo custo e relativamente fácil de se construir. Nesse sentido, a Tabela 1 apresenta os itens que compõem o dispositivo e seus preços, obtidos em janeiro de 2017.

A concepção do dispositivo MPR datalogger foi realizada de tal maneira que fosse possível medir, registrar e armazenar os dados de data, hora e pressão hidráulica, e os valores registrados deram origem a uma série temporal, que posteriormente foi avaliada sob a perspectiva da operação do presente sistema.

Tabela 1 - Componentes utilizados no dispositivo e seus respectivos preços.

\begin{tabular}{l|c}
\hline Item & Custo (R\$) \\
\hline Sensor MPX5700DP & 69,90 \\
\hline Bateria 18650 com suporte & 40,00 \\
\hline Caixa plástica & 20,00 \\
\hline Real time clock DS3231 & 20,00 \\
\hline Conjunto de engate rápido & 19,50 \\
\hline Microcontrolador ATmega328/P & 15,90 \\
\hline EEprom 24LC1024 & 15,00 \\
\hline PCB & 4,00 \\
\hline Chave liga/desliga & 1,50 \\
\hline LED & 1,00 \\
\hline Borne & 0,90 \\
\hline Capacitor de 1000 uF & 0,25 \\
\hline Resistor 220 ohms & 0,10 \\
\hline Resistor 10.000 ohms & 0,10 \\
\hline Total de custo do dispositivo & 208,15 \\
\hline
\end{tabular}


口-

Inicialmente, montou-se um protoboard para instalar o circuito e realizar o protótipo do dispositivo. As placas peripheral component interconnect (PCI) foram trabalhadas a partir da concepção de transferência térmica e corrosão química. Perfuraram-se as placas para disposição de seus componentes, tendo sido colocado o sensor de pressão internamente no ponto de coleta do dispositivo. Alocaram-se os componentes dentro de caixas plásticas identificadas. A Figura 1 demonstra as fases de desenvolvimento dos 15 equipamentos confeccionados.

\section{Software}

$\mathrm{O}$ algoritmo responsável pelo gerenciamento e funcionamento do equipamento foi desenvolvido em linguagem $\mathrm{C}++$, que é uma linguagem de programação compilada multiparadigma. $\mathrm{O}$ dispositivo foi programado para ser capaz de armazenar o dia, o mês, a hora e o minuto, nesta ordem, ao momento de cada medição da pressão. Posterior a isso, o processo era iniciado novamente. As Figuras 2 e 3 apresentam, respectivamente, o fluxograma da programação e o algoritmo em linguagem $\mathrm{C}++$ desenvolvido.

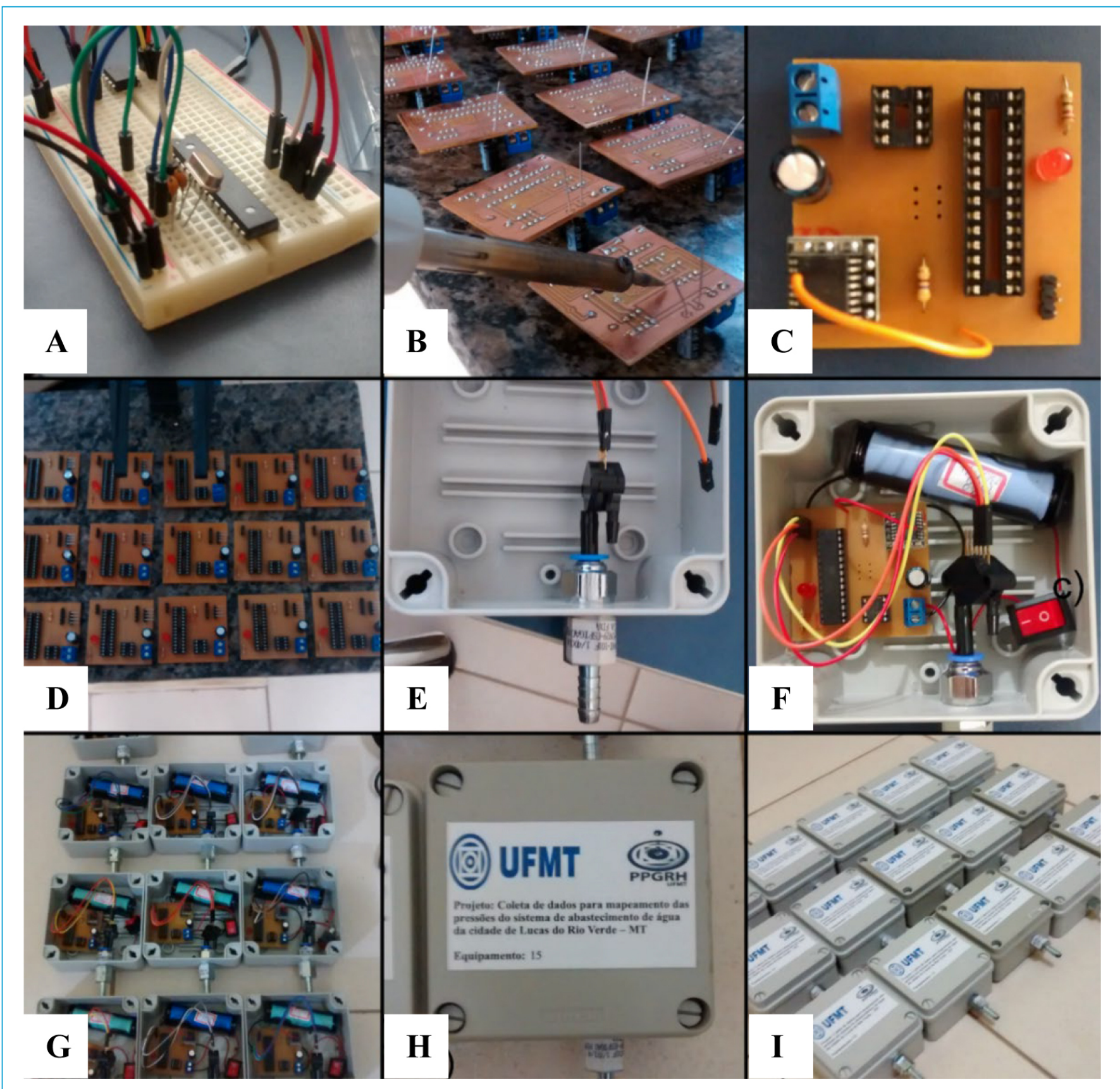

Figura 1 - MPR datalogger. (A) Protoboard; (B) PCl; (C) encaixe das peças na placa perfurada; (D) placas parcialmente montadas; (E) tomada de pressão; ( F e G) localização dos componentes dentro da caixa plástica; (H) identificação do dispositivo; (I) equipamentos prontos para instalação. 


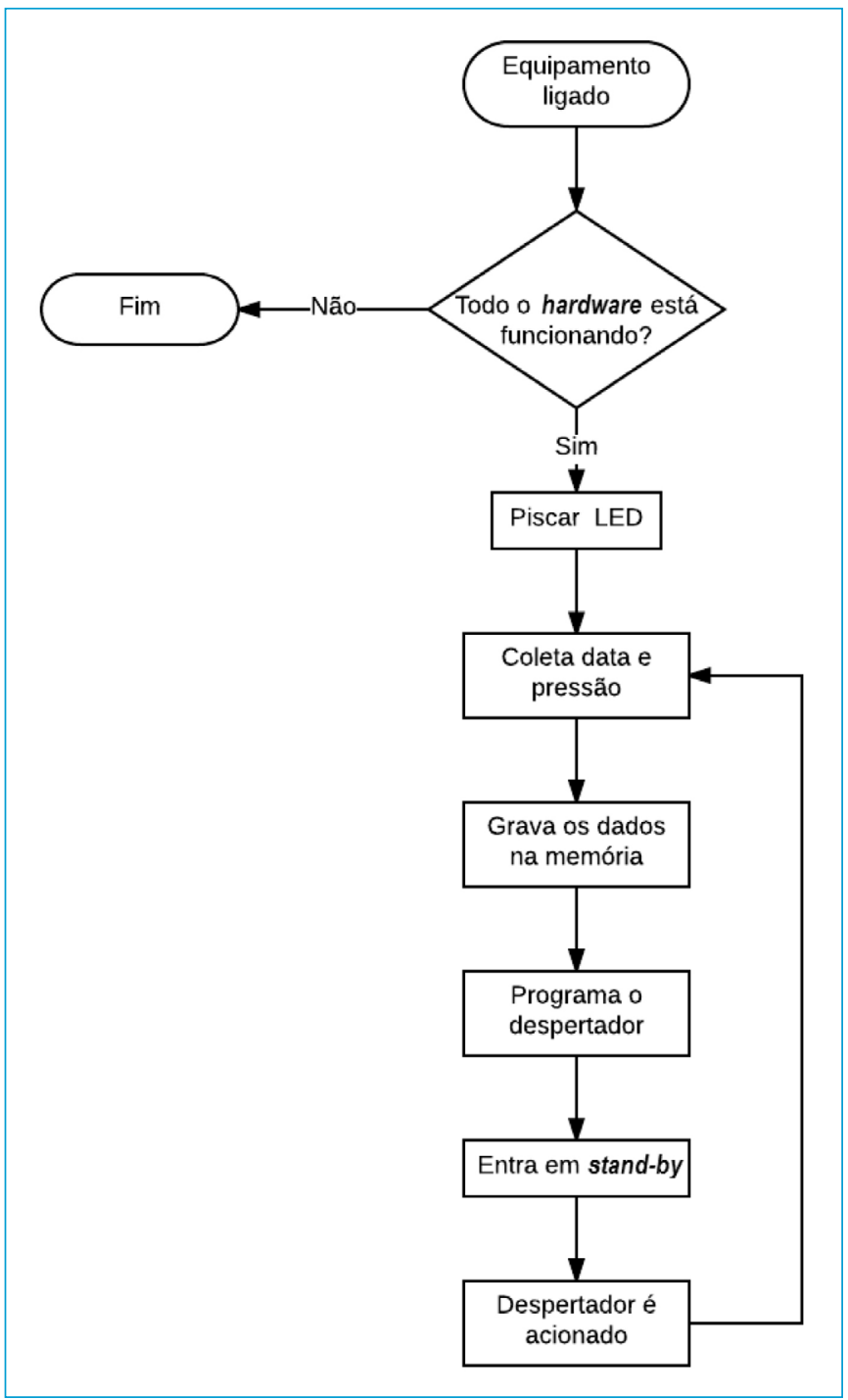

Figura 2 - Fluxograma lógico da programação desenvolvida.
Determinou-se que, quando o algoritmo fosse iniciado, o indicador de LED do equipamento piscasse para informar seu bom funcionamento, sendo realizada a primeira coleta de dados logo em seguida. Então, o alarme é acionado e colocado em stand-by para diminuir o gasto de energia. Estipulou-se que o MPR datalogger fosse religado para realizar medições em intervalo temporal de 5 minutos.

\section{Funcionamento básico do MPR datalogger}

O MPR datalogger foi desenvolvido a partir de um sensor piezoelétrico para a detecção da pressão da água abastecida. Portanto, o seu funcionamento se dava basicamente pelo mecanismo de envio de um sinal elétrico proporcional à pressão hidráulica aplicada na extremidade do sensor para o microcontrolador, que, por sua vez, convertia esse sinal em um valor inteiro, que variava entre 0 e 1.023 . $O$ valor de pressão é calculado a partir da Equação 1 e, posteriormente, armazenado na memória do sistema. A pressão medida na rede de abastecimento de água foi determinada pela pressão marcada no MPR datalogger acrescida da altura geométrica de instalação do equipamento, como apresentado na Equação 2.

$\mathrm{P}_{\text {MPR datalogger }}=0,0785 \times T_{\text {out }}-3,0741$

$\mathrm{P}_{\text {Rede }}=\mathrm{P}_{\text {MPR datalogger }}+\mathrm{Hg}$

Em que:

$\mathrm{P}_{\text {MPR datalogger }}=$ pressão medida pelo equipamento;

$\mathrm{T}_{\text {out }}=$ valor do sinal elétrico convertido entre 0 e 1.023;

$\mathrm{P}_{\text {rede }}=$ pressão da água disponível na rede de abastecimento;

$\mathrm{Hg}=$ altura geométrica entre o cavalete e a instalação do equipamento.

\section{Validação do MPR datalogger}

Um equipamento comercial medidor de pressão hidráulica foi utilizado como módulo comparativo para validar os dados obtidos pela medição do MPR datalogger. Ambos estavam conectados a uma tubulação e ligados no mesmo ponto de tomada de pressão, conforme a Figura 4. As medições dos dados e seus respectivos armazenamentos, para a validação do equipamento desenvolvido, foram realizados por 56 horas consecutivas, também em intervalo temporal de 5 minutos.

\section{Área de estudo de instalação dos MPR dataloggers e monitoramento das pressões}

Os MPR dataloggers desenvolvidos neste estudo foram instalados em 15 residências do município de Lucas do Rio Verde, localizado no estado de Mato Grosso e composto de 22 bairros e população estimada de 61.515 habitantes no ano de 2017, com 20.910 residências atendidas pelo Sistema Autônomo de Água e Esgoto (SAAE). O município está localizado a $334 \mathrm{~km}$ a norte da capital mato-grossense, tendo como via 


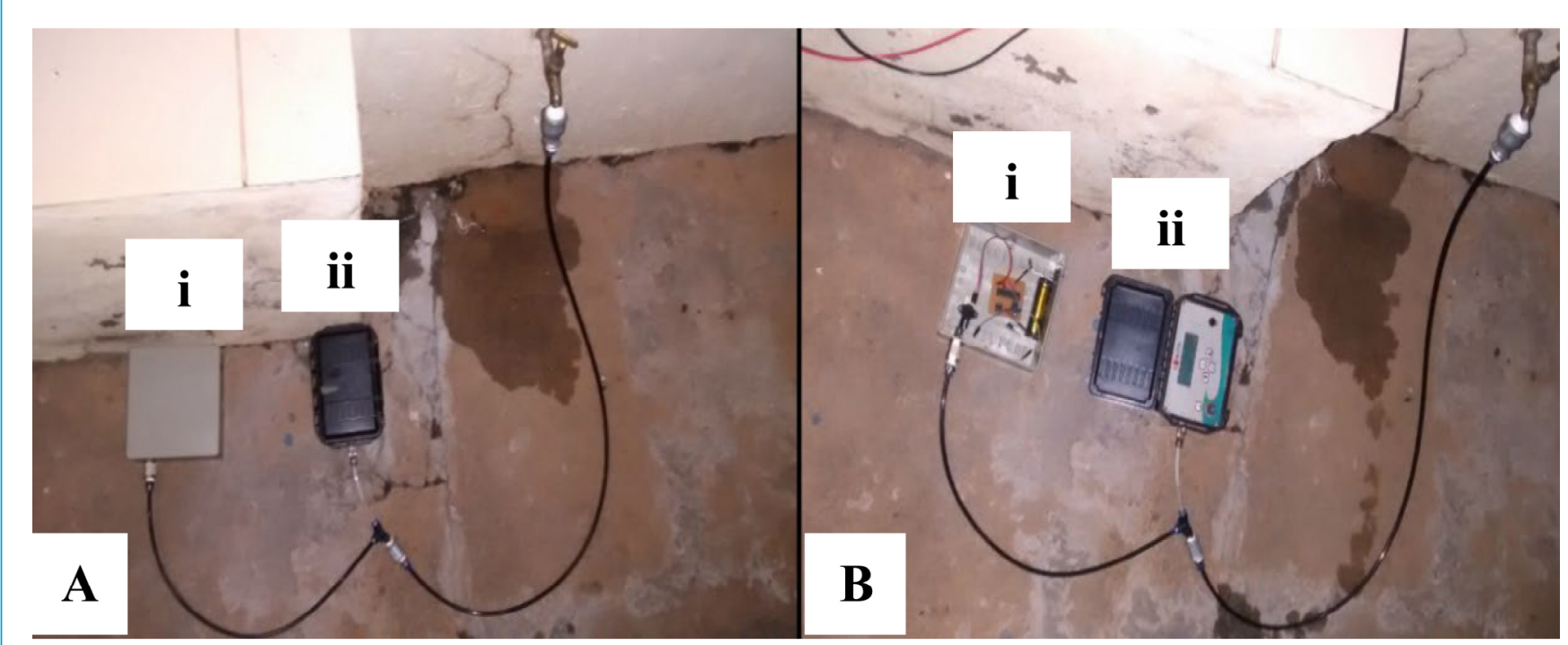

I: MPR datalogger; ii: equipamento comercial.

Figura 4 - Teste de validação da sensibilidade do equipamento. (A) Módulos fechados; (B) módulos abertos.

de aceso a BR-163, uma das principais rodovias do estado. Além disso, o município foi considerado, em 2016, a segunda cidade de pequeno porte mais desenvolvida no país (IBGE, 2017). A Figura 5 demonstra a localização da cidade onde o estudo de caso foi implementado e a divisão de seus bairros.

\section{Instalação dos MPR dataloggers nas residências para monitoramento da pressão}

A instalação dos dispositivos ocorreu de acordo com a disponibilidade de cada residência, respeitando cada particularidade. Nos locais em que não foi possível instalar os MPR dataloggers em área coberta, realizou-se a sua proteção com material plástico, para assegurar a sua integridade. Em todos os casos as instalações dos equipamentos foram realizadas acima dos cavaletes das residências, tendo sido anotadas as diferenças de cotas geométricas entre eles. As residências que participaram deste estudo possuíam uma válvula utilizada para regar o jardim, sendo esta derivada diretamente do cavalete de água. Portanto, inseriu-se um Tê para ser utilizado como ponto de tomada de pressão sem comprometer e sem modificar a dinâmica de uso das residências.

A Figura 6 apresenta a distribuição dos equipamentos MPR dataloggers, dos reservatórios e dos poços tubulares profundos, característicos do sistema de distribuição de água do município. Os pontos marcados em verde são referentes à localização dos dispositivos MPR dataloggers instalados. Os ícones vermelhos representam as unidades que bombeiam água diretamente dos poços para a rede de distribuição de água, denominados PT, enquanto os pontos azuis representam as unidades de reservação de água em pontos estratégicos.
A água bombeada dos poços possuía tratamento simplificado com cloração, que é realizada a partir de bombas dosadoras de cloro. Os reservatórios, exceto o do PT 30, possuíam pressurizadoras. Os equipamentos ficaram instalados e coletando dados de pressão dinâmica no abastecimento de água por 43 dias, durante os meses de fevereiro a abril de 2017.

\section{RESULTADOS E DISCUSSÃO}

\section{Validação do MPR datalogger}

Em consonância com as especificações dos módulos eletrônicos utilizados no desenvolvimento do presente equipamento, os MPR dataloggers possuíam faixa operacional de leitura da carga de pressão entre $0 \mathrm{e}$ 70 metros coluna d'água (mca). O componente de hardware utilizado para armazenamento de dados inferia em capacidade de até 20 mil leituras, utilizando-se memória de $125 \mathrm{~kb}$, sendo possível expandir até $512 \mathrm{~kb}$ (81.920 leituras). Salienta-se que o monitoramento das pressões foi realizado em intervalos de 5 minutos, porém o equipamento desenvolvido possuía flexibilidade de ajuste da frequência na aquisição de dados em intervalos entre 1 e 60 minutos. A utilização das baterias de lítio recarregáveis conferia autonomia de até 150 dias entre as recargas. A precisão das leituras era assegurada, além da validação entre o MPR datalogger e o equipamento comercial, pelo fabricante do componente da tomada de pressão - para o sensor MPX5700DP, a precisão é de $\pm 2,5 \%$.

O equipamento comercial utilizado para aferição das pressões coletadas e armazenadas no equipamento MPR datalogger, de acordo 


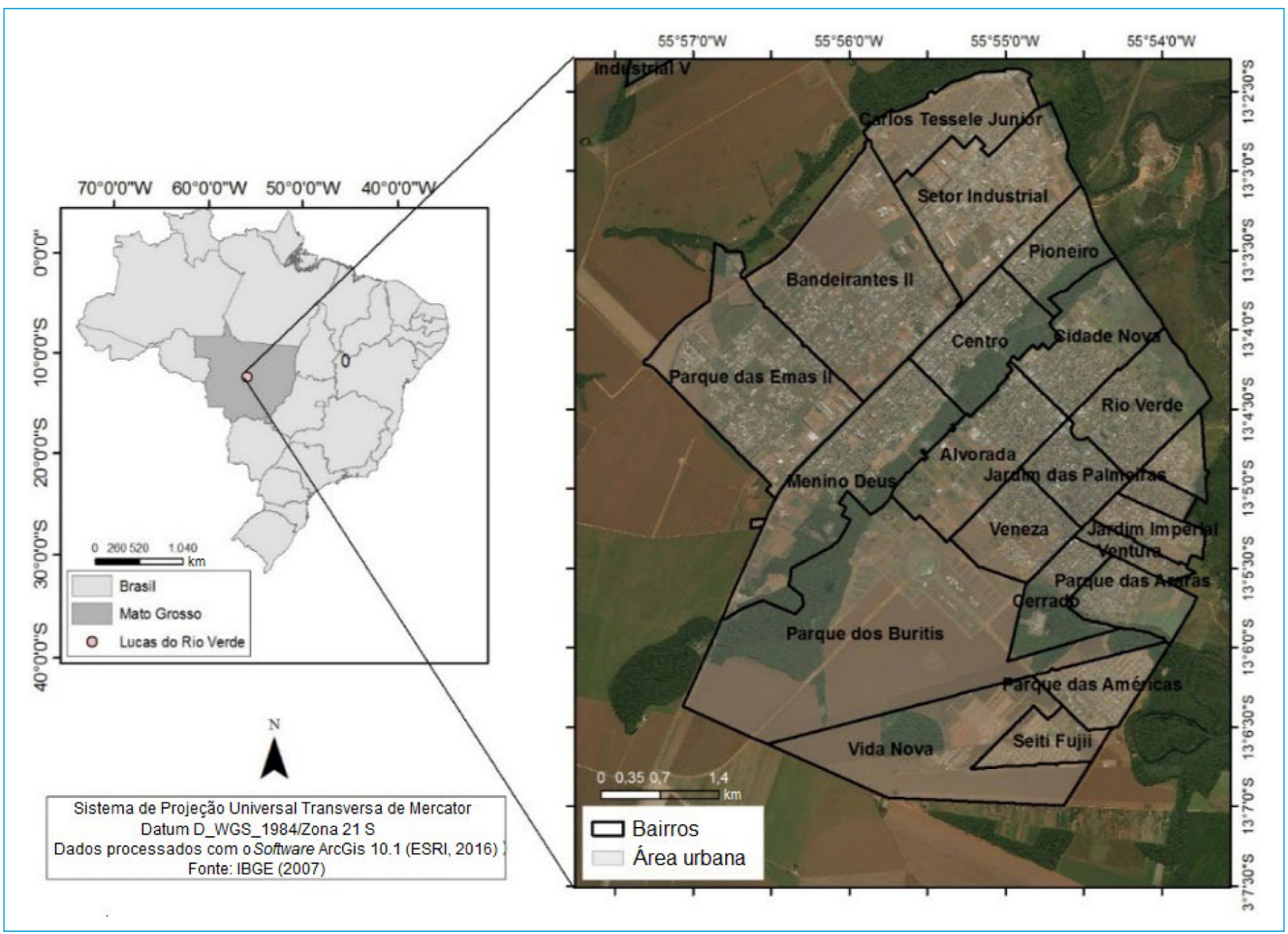

Figura 5 - Área de estudo.

com o fabricante, possuía um sensor de pressão do tipo piezo resistivo, com faixa de operação para leitura de pressão entre 0 e 200 mca. O equipamento comercial apresentava capacidade de armazenamento de $512 \mathrm{~kb}$, podendo ser expandida. A alimentação de energia ao equipamento também era realizada por uma bateria de lítio, o que conferia autonomia entre recargas de até 120 dias. Além disso, o equipamento possuía exatidão de $0,1 \% \mathrm{FS}$.

Com base na verificação das pressões medidas pelo MPR datalogger e pelo equipamento comercial foi possível constatar que os dois equipamentos funcionaram similarmente, apresentando sobreposição dos dados medidos no acompanhamento de ambos os equipamentos, conforme pode ser visto na Figura 7.

As duas quedas de pressão observadas no final das séries aferidas foram causadas pelo fechamento do registro no ponto de tomada da água. A Figura 8 demonstra a correlação dos valores de pressão da água medidos pelos dois equipamentos.

Constatou-se que as pressões medidas pelo MPR datalogger e pelo equipamento comercial possuíam uma forte correlação, com R-quadrado de 0,9986. Segundo Quinino e Reis (2011), esse coeficiente avalia a relação útil entre a variável resposta e a variável regressora em um sistema de regressão linear. Sendo assim, quanto mais a variável resposta puder ser explicada pela variável regressora, mais próximo de 1 será o valor. Além disso, o custo de aquisição do equipamento comercial, verificado em abril de 2019, é de aproximadamente R \$5.000,00. Nesse sentido, observa-se que o custo de confecção para cada MPR datalogger desenvolvido no presente trabalho é de $4,16 \%$ do valor do equipamento comercial difundido no mercado.

Sadler, Ames e Khattar (2016) desenvolveram um dispositivo de baixo custo para coleta e transmissão de temperatura e umidade, tendo sido observado funcionamento satisfatório, com desempenho de correlação entre o equipamento desenvolvido pelo grupo de pesquisadores e um equipamento comercial de $\mathrm{R}^{2}$ de 0,9963 , com custo de US $\$ 181$ para cada equipamento montado. Nessa perspectiva, os resultados obtidos nesta pesquisa inferem em funcionamento adequado do módulo para medição de pressões hidráulicas de baixo custo, o qual apresentou aferições muito próximas do equipamento comercial estabelecido no mercado. 


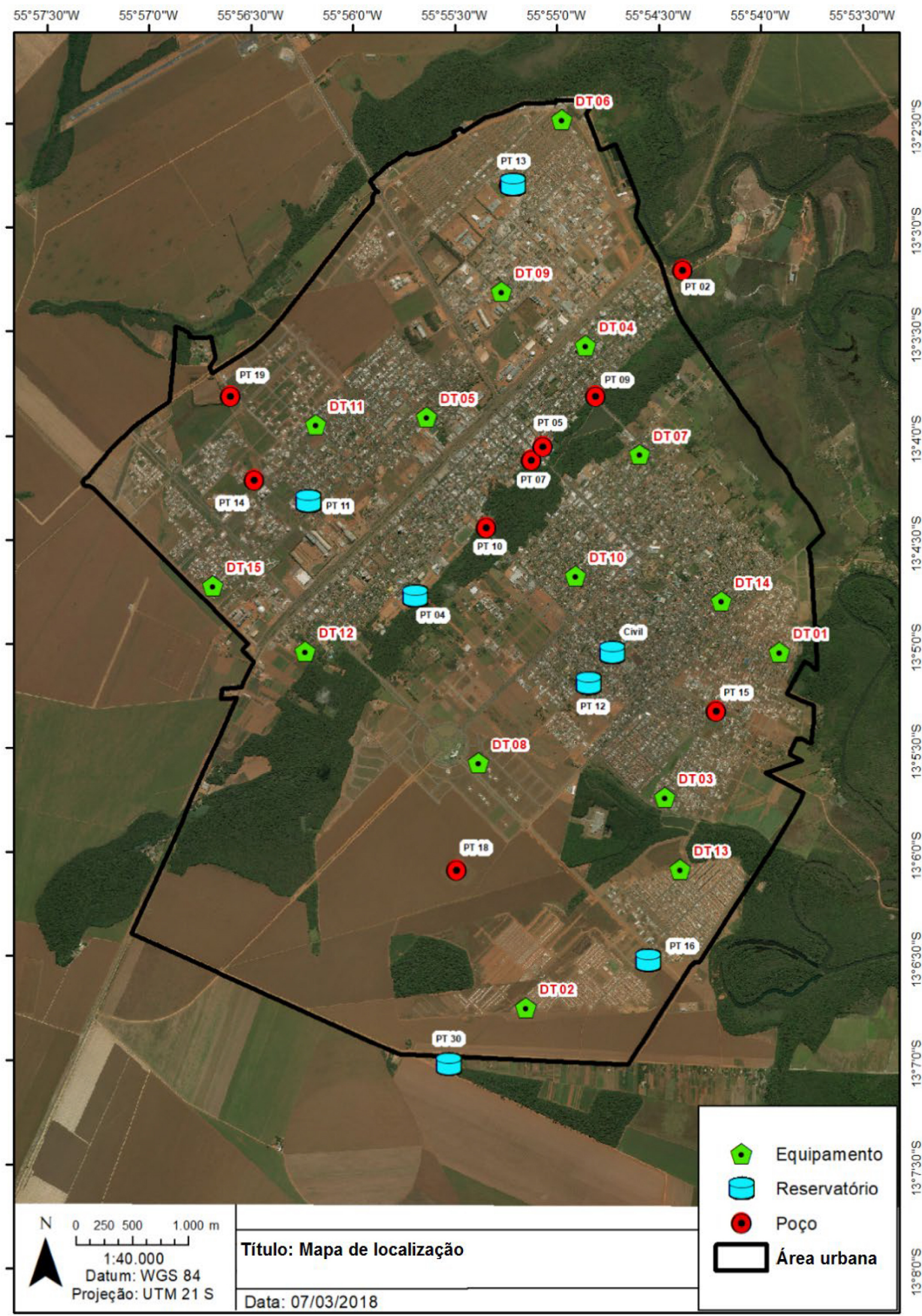

Figura 6 - Poços, reservatórios e MPR dataloggers distribuídos no município de Lucas do Rio Verde, Mato Grosso. 


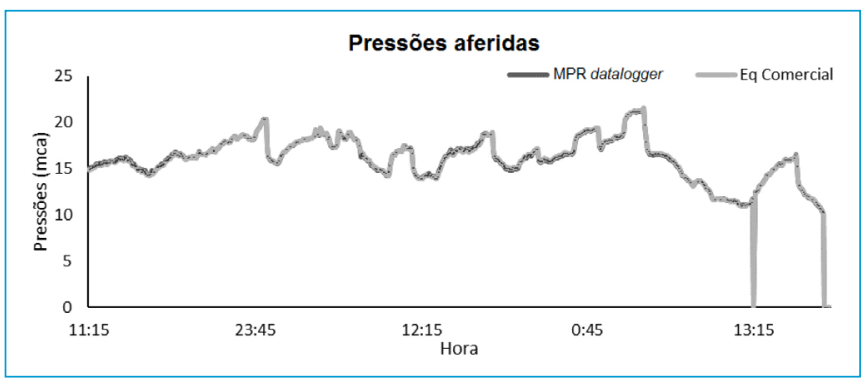

Figura 7 - Validação do MPR datalogger.

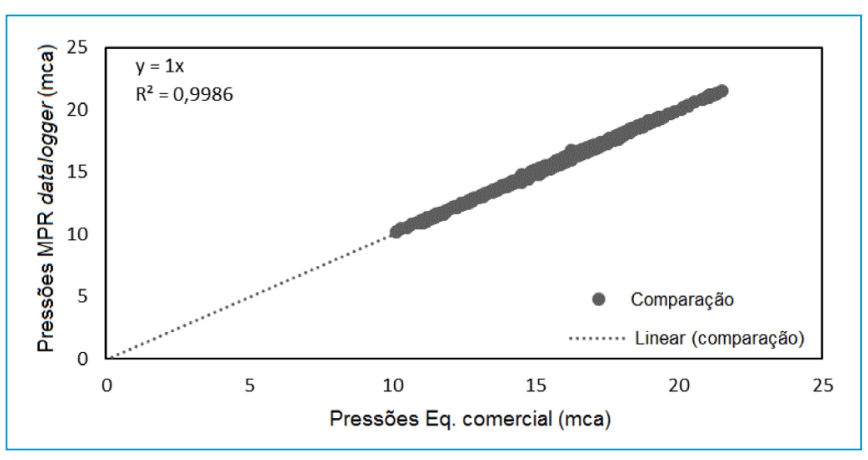

Figura 8 - Correlação das pressões aferidas pelos dois equipamentos.

\section{Acompanhamento das pressões}

Cada MPR datalogger instalado nas residências do município de Lucas do Rio Verde gerou um montante de 12.384 dados gravados na memória de seu respectivo dispositivo. As Figuras 9 e 10 apresentam o pior cenário, ou seja, o dia de menores pressões registradas dos 43 dias em relação à coleta de dados em espaço temporal de 24 horas.

A Norma Técnica Brasileira (NBR) no 12.218 (ABNT, 2017) estabelece, em seu procedimento sobre projeto de abastecimento público, que a pressão dinâmica em qualquer ponto nas tubulações de distribuição de água deve ser, no mínimo, $10 \mathrm{mca}$, e a pressão estática deve ser, no máximo, de 50 mca. Sendo assim, as concessionárias ou autarquias de abastecimento de água devem garantir essa pressão mínima de água no cavalete das residências.

Neste estudo, foi possível observar que as pressões da água que abastece as residências avaliadas não possuíam comportamento clássico das pressões hidráulicas nas redes de abastecimento, em que se espera que as pressões estejam altas à noite, o que se justifica pelo baixo consumo de água. Nesse sentido, apontam-se as particularidades do sistema de abastecimento do município, que realiza a captação de água subterrânea e o emprego de pressurizadoras, que são acionadas à medida que a demanda é requerida pelos usuários. Dessa maneira, identificou-se que cada ponto em que foi instalado um equipamento MPR datalogger era influenciado por algum poço tubular profundo, caracterizado como as principais fontes de abastecimento do sistema.

O ponto de monitoramento DT02, abastecido principalmente pelos poços PT 16 e PT 30, apresentou pressões dinâmicas mínimas satisfatórias entre $7 \mathrm{~h}$ e $20 \mathrm{~h} 30 \mathrm{~min}$. No entanto, no período noturno a pressão disponível diminuía drasticamente, fazendo com que as residências não fossem abastecidas, o que inferia na necessidade de reservatórios internos para suprir a intermitência do presente setor.

Não obstante, o DT03 apresentou pequena variação, isso porque as unidades de poços PT 15 e PT 18, que abasteciam esse ponto, funcionavam durante todo o período e, além disso, a unidade PT 16 abastecia esse setor por gravidade. Desse modo, o setor não era influenciado pelos acionamentos e desligamentos de bombas. Em adição, observou-se que essa baixa variação também pode ter sido decorrente do acionamento das pressurizadoras das unidades Civil e PT 12 no período de maior consumo.

O dispositivo MPR datalogger instalado no ponto DT06 demonstrou pressão satisfatória durante a noite, o que pode estar relacionado à localização geográfica em uma parte baixa do setor, que gerava pressão por gravidade do reservatório na unidade PT 13. Constatou-se que nos horários de alto consumo a bomba pressurizadora era acionada, sendo possível verificar no comportamento das pressões que no período das $6 \mathrm{~h}$ às $8 \mathrm{~h} 10 \mathrm{~min}$ foram efetuados dois desligamentos e três acionamentos da pressurizadora, porque com ela desligada e ligada as pressões mínima e máxima eram atingidas, respectivamente. O sistema foi estabilizado a partir das $8 \mathrm{~h} 10 \mathrm{~min}$ e às $21 \mathrm{~h} 40 \mathrm{~min}$ a pressurizadora foi novamente desligada.

No ponto amostral das pressões hidráulicas DT07 foi possível notar a influência do consumo de água no comportamento da pressão de um setor. Durante os horários de maior consumo, mesmo com o acionamento dos conjuntos moto-bombas das unidades Civil, PT 09 e PT 12, a pressão disponível reduzia em torno de 15 mca em relação ao horário de baixo consumo.

O setor onde o equipamento DT10 foi instalado era abastecido no período noturno pela unidade PT 10, que operava por 24 horas. No período de alto consumo, as unidades Civil e PT 12 auxiliavam no abastecimento: o acionamento das bombas ocorria às $6 \mathrm{~h}$ e o desligamento às $21 \mathrm{~h}$ para recuperação do nível de seus respectivos reservatórios.

O ponto de monitoramento DT12 era abastecido em horários de consumo ordinário pelo poço PT 04 e pelo PT 11, que transferia pressão ao setor por meio de uma adutora. A pressurizadora do PT 04 era acionada nos dias de alto consumo de água. O setor de instalação do equipamento DT13 era abastecido por gravidade por meio do reservatório da unidade PT 16, o que inferia em baixa variação de pressão em relação aos outros setores.

Dessa forma, os resultados demonstraram que, dos 15 pontos de instalação do equipamento MPR datalogger, 9 deles, mediante o pior 


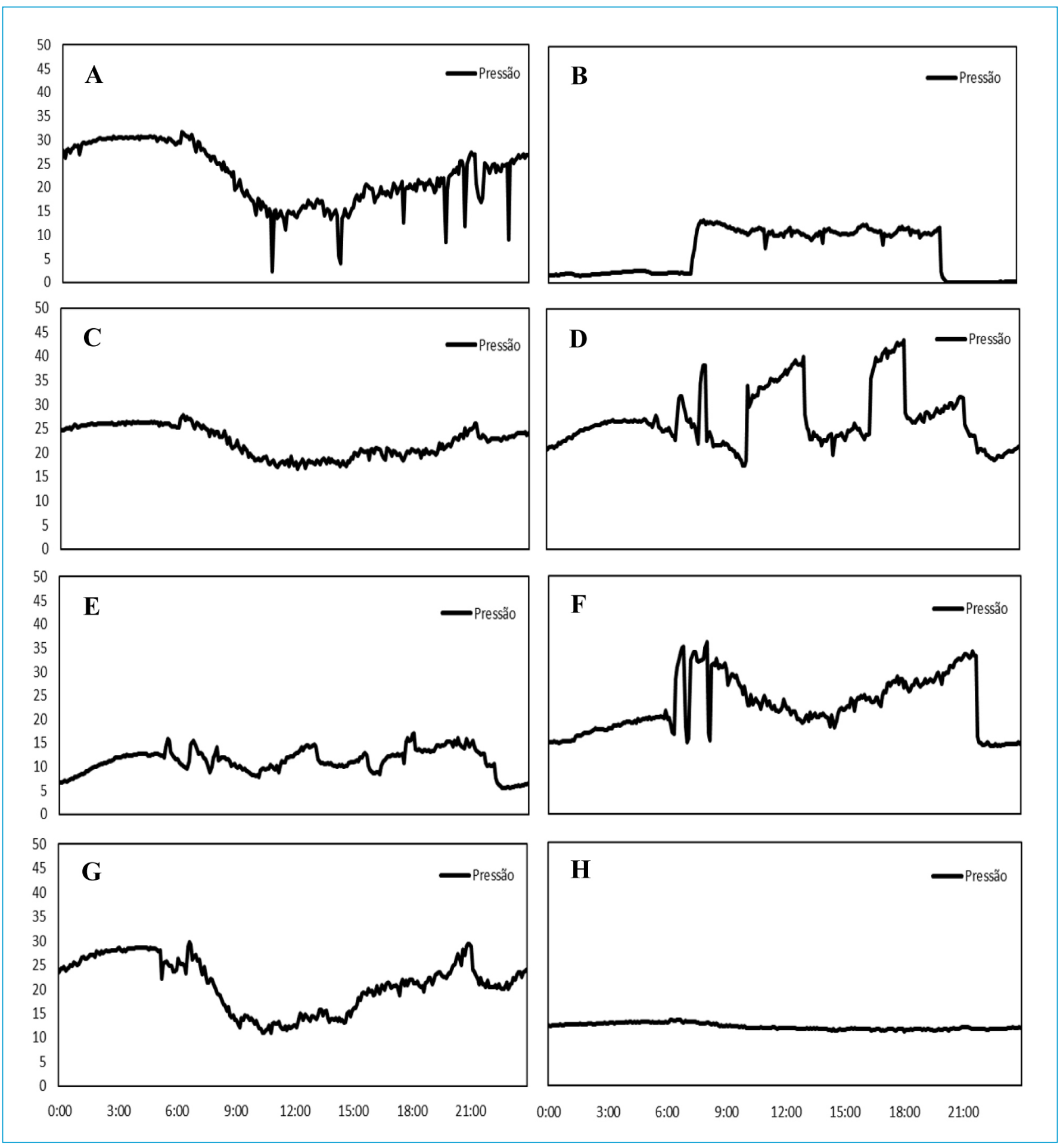

Figura 9 - Medição de pressão da água abastecida. (A) Datalogger 1 - DTO1; (B) datalogger 2 - DTO2; (C) datalogger 3 - DTO3; (D) datalogger 4 - DTO4; (E) datalogger 5 - DT05; (F) datalogger 6 - DT06; (G) datalogger 7 - DT07; (H) datalogger 8 - DTO8.

cenário de 24 horas durante os 43 dias analisados, apresentaram em algum momento pressão dinâmica menor que $10 \mathrm{mca}$, contrariando a normativa do país. Portanto, constatou-se que o monitoramento e o mapeamento das pressões medidas nos 15 pontos de coleta serviram para o entendimento da influência que as unidades de bombeamento tinham em cada setor, possibilitando a visualização clara da operação do sistema de abastecimento de água do município de Lucas do Rio Verde e as intervenções necessárias. 

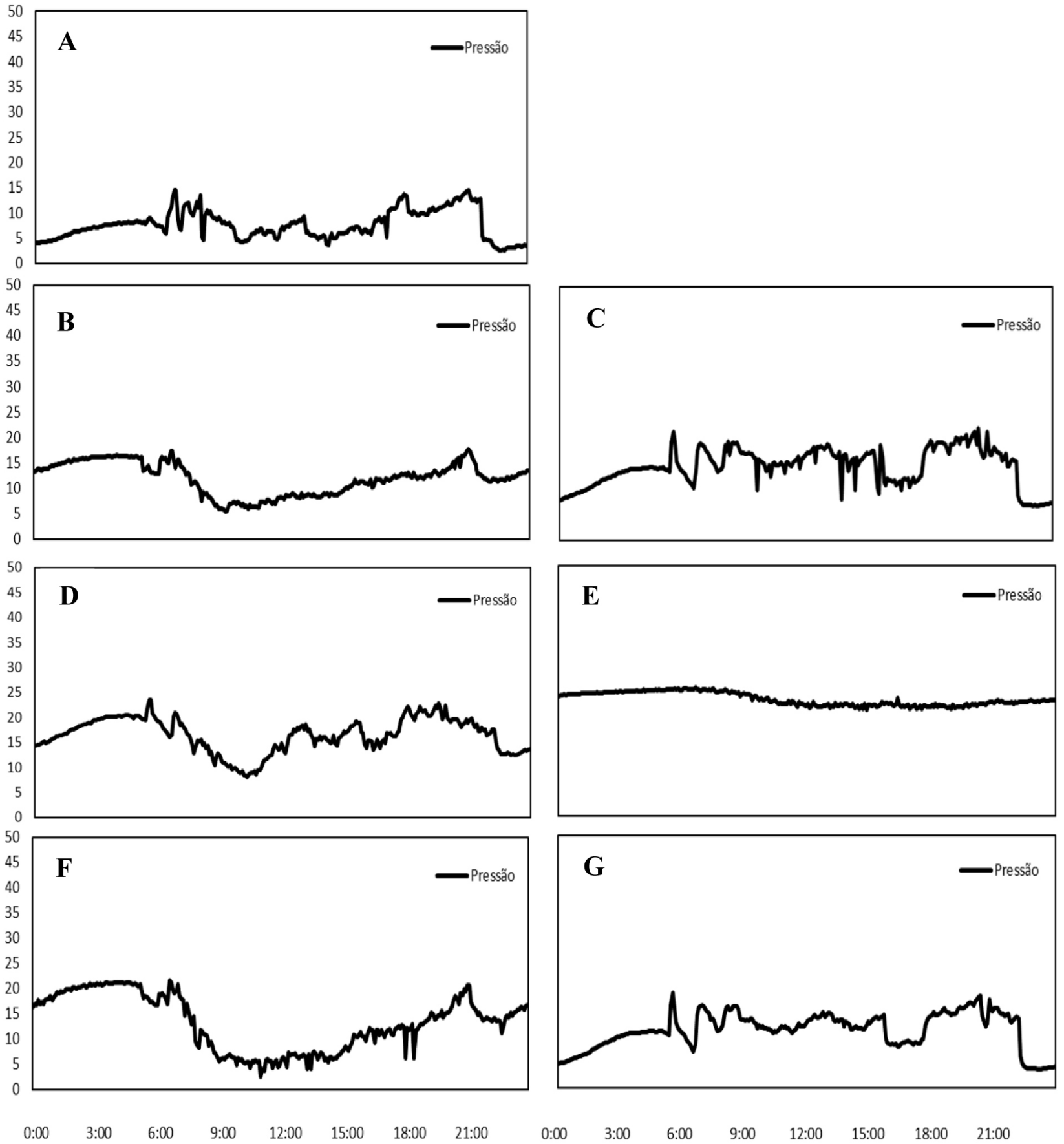

Figura 10 - Medição de pressão da água abastecida. (A) Datalogger 9 - DT09; (B) datalogger 10 - DT10; (C) datalogger 11 - DT11; (D) datalogger 12 DT12; (E) datalogger 13 - DT13; (F) datalogger 14 - DT14; (G) datalogger 15 - DT15.

\section{CONCLUSÕES}

Os dispositivos MPR datalogger, desenvolvidos para a coleta e o armazenamento das pressões de água monitoradas em diversas residências, demonstraram-se robustos e precisos, tendo sido expostos à chuva e ao sol durante os 43 dias de coleta de dados sem apresentar nenhum defeito ou anormalidade, o que corrobora com a possibilidade de implantação desses dispositivos em redes de abastecimento de água para o monitoramento das pressões hidráulicas, com baixo custo para cada dispositivo.

Além disso, os resultados do monitoramento das pressões reforçaram a necessidade de acompanhamento das condições hidráulicas do sistema de abastecimento de água sem onerosidade excessiva. As informações geradas nessa etapa do estudo possibilitaram que várias medidas fossem 
tomadas para adequar a operação nos setores que apresentavam problemas com a pressão, tais como a troca de conjuntos moto-bombas da unidade PT 13, a troca da bomba do poço da unidade PT 30, a instalação de uma adutora de $100 \mathrm{~mm}$ e a alteração na forma de operação das unidades PT 16 e PT 30, para evitar as pressões baixas no período noturno.
Para os estudos futuros, pretende-se aplicar os equipamentos desenvolvidos neste trabalho na quantificação e redução de perdas em sistemas de distribuição de água, além de executar melhorias quanto a qualidade do projeto, no sentido de produzir um invólucro em impressora 3D e adquirir placas de circuito impresso industrializadas.

\section{REFERÊNCIAS}

ASSOCIAÇÃO BRASILEIRA DE NORMAS TÉCNICAS (ABNT). (2017) NBR 12218: Projeto de rede de distribuição de água para abastecimento público - Procedimento. Rio de Janeiro: ABNT. 28 p.

BAKER, E. (2014) Open source data logger for low-cost environmental monitoring. Biodiversity Data Journal, n. 2, e1059. https://dx.doi.org/10.3897\%2FBDJ.2.e1059

BARROS FILHO, E.G.; SALVINO, L.R.; BEZERRA, S.T.M.; SALVINO, M.M.; GOMES, H.P. (2018) Intelligent system for control of water distribution networks. Water Supply, v. 18, n. 4, p. 1270-1281. https:// doi.org/10.2166/ws.2017.188

BEZERRA, S.T.M.; SILVA, S.A.; GOMES, H.P. (2012) Operational optimisation of water supply networks using a fuzzy system. Water SA, v. 38, n. 4, p. 565-572. http://dx.doi.org/10.4314/wsa.v37i4.18

CAMPISANO, A.; MODICA, C:; VETRANO, L. (2012) Calibration of Proportional Controllers for the RTC of Pressures to Reduce Leakage in Water Distribution Networks. Journal of Water Resources Planning and Management, v. 138, n. 4, 2012. https://doi. org/10.1061/(ASCE)WR.1943-5452.0000197

CLASEN, T.; FABINI, D.; BOISSON, S.; TANEJA, J.; SONG, J.; AICHINGER, E.; BUI, A.; DADASHI, S.; SCHMDT, W.P.; BURT, Z.; NELSON, K.L. (2012) Making sanitation count: developing and testing a device for assessing latrine use in low-income settings. Environmental Science Technology, v. 46, n. 6, p. 3295-3303. https://doi.org/10.1021/ es2036702

DIAS, D.M.; MARTINEZ, C.B.; LIBÂNIO, M. (2010) Avaliação do impacto da variação da renda no consumo domiciliar de água. Engenharia Sanitária e Ambiental, v. 15, n. 2, p. 155-166. http://dx.doi. org/10.1590/S1413-41522010000200008

FALLSIDE, F.; PERRY, P.F. (1975) Hierarchical optimization of a water supply network. Proceedings IEE, v. 13, n. 3, p. 98-99. https://doi. org/10.1049/iipi.1975.0021

FECAROTTA, O.; ARICÒ, C.; CARRAVETTA, A.; MARTINO, R.; RAMOS, H.M. (2015) Hydropower Potential in Water Distribution Networks: Pressure Control by PATs. Water Resources Management, v. 29, n. 3, p. 699-714. https://doi.org/10.1007/s11269-014-0836-3

FEDI, A.; FERRARI, D.; LIMA, M.; PINTUS, F.; VERSACE, C. (2013) Acronet paradigma: an open hardware project. In: OPEN WATER SYMPOSIUM, 2., 2013, Bruxelas. Anais... v. 2, n. 1, p. 7.
FORMIGA, K.T.M.; CHAUDHRY, F.H. (2008) Modelos de análise hidráulica de redes de distribuição de água considerando demanda dirigida pela pressão e vazamentos. Engenharia Sanitária e Ambiental, v. 13, n. 2, p. 153-162. https://doi.org/10.1590/ S1413-41522008000200005

GURAGAI, B.; HASHIMOTO, T.; OGUMA, K.; TAKIZAWA, S. (2O18) Data logger-based measurement of household water consumption and micro-component analysis of an intermittent water supply system. Journal of Cleaner Production, v. 197, parte 1, p. 1159-1168. https://doi. org/10.1016/j.jclepro.2018.06.198

GURUNG, T.R.; STEWART, R.A.; BEAL, C.D.; SHARMA, A.K. (2015) Smart meter enabled water end-use demand data: platform for the enhanced infrastructure planning of contemporary urban water supply networks. Journal of Cleaner Production, v. 87, p. 642-654. https://doi.org/10.1016/j.jclepro.2014.09.054

GURUNG, T.R.; STEWART, R.A.; SHARMA, A.K.; BEAL, C.D. (2014) Smart meters for enhanced network modelling and infrastructure planning. Resources, Conservation and Recycling, v. 90, p. 34-50. https://doi.org/10.1016/j.resconrec.2014.06.005

INSTITUTO BRASILEIRO DE GEOGRAFIA E ESTATISTICA (IBGE). (2017) Cidades. IBGE. Disponível em: <https://cidades.ibge.gov.br/ comparamun/compara.php?lang $=\&$ coduf $=51 \& i d t e m a=130 \& c o d v=$ v01\&search=mato-grosso\%7Cjuara\%7Csintese-das-informacoes->. Acesso em: abr. 2019

LUNDQUIST,J.D:;OTT,F.(2008)Using inexpensivetemperaturesensors to monitor the duration and heterogeneity of snowcovered areas. Water Resources Research, v. 44, n. 4. https://doi.org/10.1029/2008WR007035

MITCHELL, K.A.; CHUA, B.; SON, A. (2014) Development of fist generation in-situ pathogen detection system (Gen1-IPDS) based on NanoGene assay for near real time E. coli 0157: H7 detection. Biosensors and Bioelectronics, v. 54, 2014, p. 229-236. https://doi. org/10.1016/j.bios.2013.10.056

MOURA, G.A.; BEZERRA, S.T.M.; GOMES, H.P.; SILVA, S.A. (2018) Neural network using the Levenberg-Marquardt algorithm for optimal realtime operation of water distribution systems. Urban Water Journal, v. 15, n. 7, p. 692-699. https://doi.org/10.1080/1573062X.2018.1539503

QUININO, R.C.; REIS, E.A. (2011) Relatório Técnico: Coeficiente de Determinação R2 como Instrumento Didático para Avaliar a Utilidade de um Modelo de Regressão Linear Múltipla. Belo Horizonte: UFMG. 
SADLER, J.M.; AMES, D.P.; KHATTAR, R. (2016) A recipe for standardsbased data sharing using open source software and low-cost electronics. Journal of Hydroinformatics, v. 18, n. 2, p. 185-197. https:// doi.org/10.2166/hydro.2015.092

SARBU, I. (2016) A Study of Energy Optimisation of Urban Water Distribution Systems Using Potential Elements. Water, v. 8, n. 12, p. 593. https://doi.org/10.3390/w8120593

SOARES, A.K.; CHEUNG, P.B.; REIS, L.F.R.; SANDIM, M.P. (2004) Avaliação das perdas físicas de um setor da rede de abastecimento de água de Campo Grande-MS via modelo inverso. Engenharia Sanitária e Ambiental, v. 9. n. 4, p. 312-321. https://doi.org/10.1590/ S1413-41522004000400008

TREVATHAN, J.; JOHNSTONE, R.; CHIFLINGS, T.; ATKINSON, I.; BERGMANN, N.; READ, W.; THEISS, S.; MYERS, T.; STEVENS, T. (2012) SEMAT- the next generation of inexpensive marine environmental monitoring and measurement systems. Sensors, v. 12, n. 7, p. 9711-9748. https://dx.doi. org/10.3390\%2Fs120709711

TROJAN, F; MARÇAL, R.F.M. (2007) Study and Implement of a Specialized Monitoring System (SMS) to Orient Decisions and Reduce Losses in the Public Water Supply Systems. In: INTERNATIONAL CONFERENCE ON INNOVATIVE COMPUTING, INFORMATION AND CONTROL, 2., 2007. Anais... Disponível em: <https://doi.org/10.1109/ICICIC.2007.545>. Acesso em: abr. 2019.

TSUTIYA, M.T. (2006) Abastecimento de água. 3a ed. São Paulo: Departamento de Engenharia Hidráulica Sanitária da Escola Politécnica da Universidade de São Paulo. 643 p.

WILLIS, R.M.; STEWART, R.A.; GIURCO, D.P.; TALEBPOUR, M.R.; MOUSAVINEJAD, A. (2013) End Use water consumption in households: impact of socio-demographic factors and efficient devices. Journal of Cleaner Production, v. 60, p. 107-115. https://doi. org/10.1016/j.jclepro.2011.08.006 